\title{
Impact of Helicobacter pylori-related Metabolic Syndrome and Gastroesophageal Reflux Disease on the Risk of Acute Myocardial Infarction
}

TO THE EDITOR: In their recent study, Eisa et $\mathrm{al}^{1}$ concluded that gastroesophageal reflux disease (GERD) with concomitant metabolic syndrome (MetS) parameters is a risk factor of acute myocardial infarction (AMI) though this risk may be clinically insignificant.

In this regard, there is increasing evidence for association between Helicobacter pylori infection ( $\mathrm{Hp}-\mathrm{I})$ and insulin resistance (IR) or MetS and related morbidity, including GERD and cardiovascular disease (CVD); ${ }^{2,3}$ the prevalence of MetS is higher in $\mathrm{Hp}$ positive people; ${ }^{2} \mathrm{Hp}$-linked MetS is a risk factor of GERD and its eradication exhibits a positive effect against GERD in certain populations; ${ }^{3} \mathrm{AMI}$, a potentially fatal CVD complication, is closely linked with $\mathrm{MetS} ;{ }^{2}$ and $\mathrm{Hp}$ is a risk for acute coronary syndrome (ACS) including $\mathrm{AMI},{ }^{2}$ thus further investigation is warranted to estimate whether $H p$ eradication affects AMI occurrence.

Specifically, both $\mathrm{Hp}$-I and MetS are highly prevalent worldwide and epidemiological studies as well as meta-analyses have shown that obesity induces inflammation (especially abdominal, visceral obesity) and drives to MetS, thereby being an indirect risk factor for GERD. ${ }^{3}$ In this respect, the conventional claim that declining $H p$ prevalence has led to a rise in GERD requires to be better studied since, for instance, the current global prevalence of $\mathrm{Hp}$-I varies from $39.9 \%$ to $84.2 \%$ whereas the comparable picture for GERD is quite less varying from $2.5 \%$ to $51.2 \% .^{4}$ Moreover, several data indicate that $\mathrm{Hp}$ may contribute to GERD pathogenesis by several mechanisms and its eradication results in adequate control of GERD symptoms and improves esophagitis. ${ }^{3,5}$

A recent meta-analysis also indicated that $\mathrm{Hp}$-I increases the risk of CVD adverse events, particularly AMI; ${ }^{6}$ there is a link be- tween $\mathrm{Hp}$-related CagA cytotoxin and ACS and the odds ratio of AMI is twice as greater in $\mathrm{Hp}$-positive patients. Likewise, MetS is a major risk factor for $\mathrm{AMI}^{7}$ increases the risk of $\mathrm{CVD}$ adverse events more than 2-fold, whereas its recovery significantly decreases the risk for major adverse cardiovascular events including AMI. Moreover, $\mathrm{Hp}$-I is an independent risk factor for atrial fibrillation $(\mathrm{AF}),{ }^{8}$ which remains a frequent arrhythmia in AMI and is closely linked with augmented subsequent cardiovascular mortality; coronary artery disease is a risk factor for AF and coronary embolism due to AF is a cause of AMI; and Hp-related non-alcoholic fatty liver disease, the hepatic component of MetS, is a risk of $\mathrm{AF}^{8} \mathrm{Fi}^{-}$ nally, $\mathrm{Hp}$-I-related MetS may contribute to the pathophysiology of CVD including AMI by several mechanisms, ${ }^{9,10}$ thereby signifying eradication therapy as AMI prevention strategy.

Jannis Kountouras, ${ }^{1 *}$ Apostolis Papaefthymiou, ${ }^{1,2,3}$
Michael Doulberis, ${ }^{1,3,4}$ Stergios A Polyzos, ${ }^{3}$ Christos Zavos, ${ }^{1}{ }^{1,5}$
Evangelos Kazakos, ${ }^{1,5}$ Sofia K Tzika, ${ }^{1}$ Elisabeth Vardaka, ${ }^{1,6}$
Christos Liatsos, ${ }^{7}$ and Panagiotis Katsinelos ${ }^{1}$
${ }^{1}$ Department of Medicine, Second Medical Clinic, Aristotle University
of Thessaloniki, Ippokration Hospital, Thessaloniki, Macedonia,
Greece; ${ }^{2}$ Department of Gastroenterology, University Hospital of
Larisa, Mezourlo, Larisa, Greece; ${ }^{3}$ First Laboratory of Pharmacology,
Aristotle University of Thessaloniki, Thessaloniki, Macedonia, Greece;
${ }^{4}$ Division of Gastroenterology and Hepatology, Medical University
Department, Kantonsspital Aarau, Aarau, Switzerland; ${ }^{5}$ Faculty of
Midwifery, School of Health Sciences, University of Western Macedonia,
Greece; ${ }^{6}$ Department of Nutritional Sciences and Dietetics, School of
Health Sciences, International Hellenic University, Alexander Campus,
Thessaloniki, Macedonia, Greece; and ${ }^{7}$ Department of Gastroenterology,
401 General Military Hospital of Athens, Athens, Greece
myocardial infarction in patients with gastroesophageal reflux disease. J


Neurogastroenterol Motil 2020;26:471-476.

2. Franceschi F, Gasbarrini A, Polyzos SA, Kountouras J. Extragastric diseases and Helicobacter pylori. Helicobacter 2015;20:40-46.

3. Kountouras J, Polyzos SA, Doulberis M, et al. Potential impact of Helicobacter pylori-related metabolic syndrome on upper and lower gastrointestinal tract oncogenesis. Metabolism 2018;87:18-24.

4. Kountouras J, Doulberis M, Papaefthymiou A, et al. A perspective on risk factors for esophageal adenocarcinoma: emphasis on Helicobacter pylori infection. Ann N Y Acad Sci 2019;1452:12-17.

5. Kountouras J, Zavos C, Chatzopoulos D. H pylori infection and reflux oesophagitis. Gut 2004;53:912.

6. Wang B, Yu M, Zhang R, Chen S, Xi Y, Duan G. A meta-analysis of the association between Helicobacter pylori infection and risk of atherosclerotic cardiovascular disease. Helicobacter 2020:e12761.

7. Sarkar S, Paul BK, Chakraborty PK, et al. Association between metabolic syndrome and acute myocardial infarction (AMI). Mymensingh Med J 2016;25:628-634.
8. Kountouras J, Doulberis M, Papaefthymiou A, Polyzos SA. Impact of Helicobacter pylori-linked metabolic syndrome on non-alcoholic fatty liver disease and its connected atrial fibrillation risk. Liver Int 2020;40:2036-2037.

9. Kountouras J, Polyzos SA, Katsinelos P, et al. Cardio-cerebrovascular disease and Helicobacter pylori-related metabolic syndrome: we consider eradication therapy as a potential cardio-cerebrovascular prevention strategy. Int J Cardiol 2017;229:17-18.

10. Boziki M, Grigoriadis N, Doulberis M, Papaefthymiou A, Polyzos SA, Kountouras J. Potential impact of Helicobacter pylori-related Galectin-3 on chronic kidney, cardiovascular and brain disorders in decompensated cirrhosis. Dig Liver Dis 2020;52:121-123.

\section{Financial support: None.}

Conflicts of interest: None.

Author contributions: All the authors have helped in the drafting and writing of the manuscript. 